GROSVENOR (Ian), LAWN (Martin), ROUSMANIERE (Kate) (eds). - Silences \& Images. The Social History of the Classroom

New York : Peter Lang, 1999. - 275 p.

Marie-Claude Derouet-Besson

\title{
OpenEdition
}

Journals

Édition électronique

URL : https://journals.openedition.org/histoire-education/935

DOI : 10.4000/histoire-education.935

ISSN : 2102-5452

Éditeur

ENS Éditions

Édition imprimée

Date de publication : 1 janvier 2002

Pagination : 141-145

ISBN : 2-7342-0903-9

ISSN : 0221-6280

Référence électronique

Marie-Claude Derouet-Besson, « GROSVENOR (Ian), LAWN (Martin), ROUSMANIERE (Kate) (eds). Silences \& Images. The Social History of the Classroom », Histoire de l'éducation [En ligne], 93 | 2002, mis en ligne le 15 janvier 2009, consulté le 20 mai 2021. URL : http://journals.openedition.org/histoireeducation/935; DOI : https://doi.org/10.4000/histoire-education.935

Ce document a été généré automatiquement le 20 mai 2021.

(c) Tous droits réservés 


\section{GROSVENOR (Ian), LAWN (Martin), ROUSMANIERE (Kate) (eds). - Silences \& Images. The Social History of the Classroom}

New York : Peter Lang, 1999. - 275 p.

Marie-Claude Derouet-Besson

\section{RÉFÉRENCE}

GROSVENOR (Ian), LAWN (Martin), ROUSMANIERE (Kate) (eds). - Silences \& Images. The

Social History of the Classroom. - New York : Peter Lang, 1999. - 275 p.

1 Le silence des historiens sur les pratiques, le sens et la culture de la classe a décidé les auteurs à organiser, en 1995 et 1996, deux rencontres internationales (États-Unis, Belgique, Royaume-Uni, Suède, Pays-Bas, Canada) consacrées aux routines et aux activités de la classe. Comment rendre compte, à partir de sources hétérogènes, images et photographies, récits autobiographiques, journaux de bord, lettres d'enseignants ou d'élèves, plans de locaux scolaires, manuels, etc. -, d'un objet luimême composite tenant à la fois des éléments matériels de la salle de classe, des pratiques pédagogiques, des exigences curriculaires, des dynamiques et des expériences des acteurs, passés ou présents, etc. ? Quelle méthode définir pour aborder, en historiens, une facette de l'éducation, souvent voilée par les souvenirs et les émotions personnels, peut-être plus travaillée en thérapie psychanalytique qu'en histoire? L'ouvrage cherche à innover en explorant des modes de travail, des méthodes, des théories, en visant davantage l'ouverture de perspectives nouvelles que l'établissement de preuves absolues. À côté des différences d'époques, d'architectures, de nationalités, il s'agit de comparer différentes conceptualisations des classes et différentes façons de les interroger. Il s'agit de ne pas gommer les tensions, les 
articulations, les résonances, mais de les confronter pour atteindre la multiplicité des sens par celle des approches. Le livre, qui se veut animé de l'esprit novateur des rencontres, répertorie dans une première partie les questions émergentes et présente dans la deuxième partie sept études de cas illustrant la nouvelle histoire sociale de la classe. La troisième partie, "Questions émergentes/réponses à trouver ", se limite à la publication de douze photographies de classe ou de pages de manuels quasiment sans commentaire.

2 La première partie s'ouvre sur une revue de l'histoire de la classe et de son évolution (Dams, Marc Depaepe, Frank Simon, pp. 15-46). L'interrogation sur le climat de la classe s'est constituée en champ spécialisé dans les années soixante pour constater la difficulté des historiens à rendre compte des perceptions des élèves à l'école. Que pouvons-nous savoir? Que voulons-nous savoir? Quelles sont les raisons qui nous poussent à faire des recherches sur l'histoire de la classe? (p. 17). Répondre, c'est, pour les auteurs, concevoir l'histoire de la classe comme l'ouverture de la boîte noire où s'articulent les prescriptions curriculaires, pédagogiques, etc., et les réalités humaines et sociales, où se construisent les effets des décisions et des pratiques. Répondre, c'est prendre conscience des limites de l'investigation et des précautions qu'elle demande. Plus que d'élaborer des certitudes, il s'agit de repérer "des effets possibles de l'école » (p. 22), comme ceux de la peur ou du silence sur la socialisation. Les auteurs étayent leur propos par des exemples, surtout belges, pris entre 1880 et 1960. L'augmentation de l'intérêt des pédagogues pour les effets du climat de la classe doit être étudiée mais leurs textes et témoignages exigent une analyse rigoureuse car la confusion entre l'intention, la pratique et le résultat y est fréquente. L'évolution des rapports entre l'école et son environnement est devenue objet d'enquête. De l'intrusion dans la classe d'éléments extérieurs (la foire du village ou Internet) jusqu'à leur maitrise par l'enseignant, les modes de gestion du rapport au monde varient, même si les argumentations perdurent d'une époque à l'autre et que l'école reste une institution à part (p. 46). L'évaluation aussi a changé, englobant aujourd'hui différents aspects de la personnalité de l'élève, au-delà de son instruction proprement dite, et bouleversant les interactions entre enseignant et élèves. Du châtiment corporel à la discussion, de l'ordre à la négociation, le quotidien de l'école s'est à l'évidence transformé.

3 À propos de l'évolution curriculaire suédoise, l'article de Johansson (pp. 49-62) plaide pour un recentrage sur l'école "réelle», dégagée des stéréotypes marxisants ou structuralistes, pour une histoire vécue du curriculum. L'histoire de l'éducation ne doit pas se confondre avec la recherche des conditions de la reproduction. L'histoire du curriculum doit replacer l'être humain au centre de ses préoccupations et choisir résolument des approches post-modernes et post-structuralistes. Briser le silence de la classe, c'est écouter toutes les voix, y compris les plus faibles, tous les témoignages, y compris ceux qui paraissent très indirects, sous toutes leurs formes. L'auteur dresse l'inventaire des sources, très diverses qu'elle a utilisées, en particulier pour l'étude des cours de tempérance. C'est aussi s'interroger sur les silences de ces sources et les contradictions qu'elles révèlent, par exemple, entre le refoulement des initiatives des élèves en classe et leur inventivité dès qu'ils en sortent.

4 Martin Lawn traite de ce qu'il nomme la technologie de la classe (pp. 65-82), c'est-à-dire la combinaison d'éléments matériels et humains qui constituent l'action d'enseignement. Insistant sur les réajustements permanents des personnes et des objets, il amorce une bibliographie commentée concernant le mobilier, les matériels, la 
forme de la salle, celle du bâtiment scolaire, et leur impossible séparation de l'action pédagogique dans la classe et du sens, en particulier social, de cette action. Que tirer des photographies? Ian Grosvenor (pp. 86-104) propose une méthode d'analyse où la critique historique est prépondérante. Un cliché mérite autant d'analyse qu'une autre source et Grosvenor le démontre grâce au schéma d'observation détaillé de photographies de classe qu'il présente. Tout doit faire question et permettre une véritable déconstruction de l'image produite par le photographe, qu'il soit professionnel ou familial. Un dernier type de source, les lettres, est abordé par Harry Smaller (pp. 107-120) qui commente la courte lettre qu'un administrateur local canadien adresse, en décembre 1865 , à l'autorité provinciale sur les obligations du nettoyage de la salle de classe par le maître. Au-delà d'éléments descriptifs, c'est le témoignage sur la professionnalisation du métier d'enseignant et sur les relations entre élèves et maître qui fait tout l'intérêt de cette lecture et montre la richesse de ce type de sources.

5 Sans classement ni cohérence évidents, ni nette rupture de point de vue avec les questions émergentes de la première partie, la seconde partie cherche des réponses à travers sept études de cas de la nouvelle histoire sociale de la classe.

6 Pour Philip Gardner (pp. 125-144), comprendre la classe, c'est comprendre un personnage central, le maître, qu'il étudie au Royaume-Uni de 1903 à 1945. Pour Valinda Littlefield (pp. 147-161), reconstruire l'histoire d'une classe afro-américaine, c'est retrouver, à un moment et dans un lieu particuliers, la complexité des valeurs d'une société, politiques, religieuses, raciales, de gender, culturelles, grâce à une analyse fine de témoignages, d'anecdotes sur des pratiques ludiques en classe. Pour Lars-Göran Högman (pp. 163-179), l'étude de la vie d'Éva, de 1982 à 1995, lui permet d'explorer l'importance de la classe dans la construction identitaire d'une enseignante, devenue responsable du développement pédagogique dans sa ville suédoise après avoir été professeur. Pour David Vincent (pp. 181-197), l'histoire de l'apprentissage de la lecture, fondée sur l'étude de manuels et de méthodes anglais, permet d'éclairer le débat actuel en dégageant deux clans : celui qui, depuis le xvII ${ }^{\mathrm{e}}$ siècle, s'affronte sur les techniques et celui, plus récent, qui met en avant l'importance du sens de la lecture pour l'apprenant. Jo Anne Preston (pp. 199-216) conforte le dossier de l'intérêt des sources épistolaires à propos de lettres d'enseignantes de Nouvelle-Angleterre au xix ${ }^{e}$ siècle. Elles fourmillent d'informations sur les équipements, les approches pédagogiques, la vie de la classe et les relations humaines et sociales qui l'animent, les relations avec la hiérarchie, le plus souvent masculine. La correspondance des enseignantes et leurs écrits personnels constituent donc une source majeure, que l'auteur invite à répertorier et à analyser. Ann Short Chirhart (pp. 220-234) analyse la culture professionnelle d'enseignantes afro-américaines et blanches en Géorgie de 1920 à 1950. Leur foi dans les bénéfices de l'éducation conduisit les unes et les autres à accroître les enseignements fondamentaux et à faire reculer les références aux valeurs communautaires et culturelles de l'économie et de la société cotonnières. Kate Rousmaniere (pp. 238-255) s'intéresse à Margaret Haley qui, au tournant du $\mathrm{XIX}^{\mathrm{e}}$ et du $\mathrm{xx}^{\mathrm{e}}$ siècles, quitta sa classe de Chicago après seize ans d'exercice pour diriger le plus grand syndicat d'enseignants des ÉtatsUnis. La biographie du personnage lui permet de relier entre elles différentes facettes, personnelles ou institutionnelles, d'un individu en s'appuyant sur tous les types de sources disponibles. Loin de l'historien traditionnel, le biographe est une sorte de 
chiffonnier qui récupère tout ce qui peut servir à regarder l'expérience enseignante dans la durée.

7 La réduction de la troisième partie à une série de clichés photographiques accentue l'impression de travail en train de se faire que revendique l'ouvrage.

Silences \& Images s'inscrit ainsi dans plusieurs courants contemporains, la montée de l'histoire patrimoniale, le mouvement post-critique, la réhabilitation de l'émotion comme objet d'étude. Il s'agit, à la fois et plus ou moins selon les contributions, de s'écarter d'un positivisme trop réducteur, de chercher des racines plus locales à l'action et d'en explorer les conditions en s'attardant autant, peut-être davantage, sur les écarts que sur les constantes, en traquant les sens différents qu'elle peut prendre selon les personnes, les environnements, etc., en ayant moins peur d'inclure les émotions, les sentiments dans le champ des analyses, tout en restant aussi rigoureux que possible. Tout travail sur un objet positif doit être accompagné d'un regard sur la construction même du travail. La méthode de production du livre est elle-même révélatrice de cette attitude à la fois téméraire - s'attaquer différemment à l'histoire sociale de la classe - et contrôlée puisque des réunions internationales permettent aux chercheurs de comparer leurs objets, leurs regards et, surtout, de s'assurer qu'il peut être construit autrement, qu'ils ne se sont pas enfermés dans une problématique ou une épistémologie. De ce point de vue, le livre ne rend pas assez compte des productions de ces réunions et retrouve les sentiers battus de la juxtaposition des interventions.

9 L'intérêt principal du livre, outre l'ouverture sur la bibliographie anglo-saxonne des années quatre-vingt et quatre-vingt-dix, nous semble être ailleurs, dans une proximité d'intérêt avec les sociologues. L'approche de la classe privilégiée dans l'ouvrage aborde ce que les sociologues appellent la situation d'enseignement. Comme en sociologie de l'éducation, il s'agit de pénétrer une complexité, celle de la classe, et de comprendre comment les acteurs, chacun à leur manière, avec leurs références, leurs modes d'action, tentent de la réduire pour y trouver leur place et la vivre. La perspective a changé. Il s'agit de dégager ce qui fait sens pour ceux qui se retrouvent directement engagés dans la situation de la classe, de l'établissement, ou qui participent, de près ou de plus loin, comme les parents, à ce qui s'y déroule. Cette question du sens de l'action pour les acteurs implique que des sources, écartées dans les approches antérieures, deviennent centrales. Plus que l'attention accordée aux archives personnelles, déjà largement développée par les historiens francophones, c'est la combinaison de sources hétérogènes autour de l'investigation d'un même objet qui constitue l'intérêt de l'ouvrage, même si cet objet reste largement à construire. 